\title{
Definition of numerical models for rapid product development
}

\author{
A. Bernard, M. Véron \\ Research Center for Automatic Control of Nancy \\ BP 239 - 54506 - Vandoeuvre Cedex - France \\ Tel : + 33 (0) 383912729 ; Fax : + 33 (0) 383912390 ; e- \\ mail : bernard@cran.u-nancy.fr
}

\begin{abstract}
The initial stage of design needs CAD software for direct numerical and virtual definition, or requires a man-made model for direct experimental validation on a 3D physical model (ergonomics, aesthetics). After this validation phase the physical model is digitized and a numerical model is obtained from point clouds using sensors and special reverse engineering software. Most often, these two complementary techniques (CAD and scanning) are both used for the design of the complete object. After the complete definition of a numerical model the next step is the manufacturing of functional prototypes, whose characteristics are very close to those of the final industrial product (material, color, manufacturing process,). This demands the obtention of temporary tools (molds) which are used for small batch production (five to twenty parts), and the production of parts using layer manufacturing machines which are integrated as lost models during processes like investment casting. These different stages, from the idea to the functional prototypes, highlight the necessity of data integration, not only for part models and simulation results, but also for intermediate parts and tools definition. The final numerical reference model has to be coherent with the different intermediate numerical models (point clouds, STL files, tool models, etc.). In order to assume such objectives, we propose some first basic elements of a representation model for prototypes and temporary tools. This aspect corresponds to different tasks of the IMS-RPD project. The parametric aspect is discussed and its interest is shown for families of parts (tiles, jewels, etc.). The different links and types of elements of the models are proposed and illustrated by an industrial example.
\end{abstract}

\section{Key words}

Rapid product development, data integration, rapid prototyping, reverse engineering 


\section{INTRODUCTION}

One of the main factors in achieving primacy in a continuously changing market is the ability to rapidly introduce new products.

The time is past when it was enough to have the best product at the lowest cost and with the best quality/price ratio. If an industrial firm is to satisfy the requirements of its customers and overcome the competition, it must be able to control delivery times (increasingly short), quality (increasingly high) and costs (increasingly low).

Mastering these three aspects with their apparently contradictory constraints involves introducing approaches of the "concurrent engineering" type. One of the advantages of this kind of approach is that several design alternatives can be tested and simulated within an acceptable time.

Against this background, where the processes of designing and industrializing a new product take place in parallel, the use of physical models to validate an overall product design or an industrialization process takes on increased importance. The virtual model used in $\mathrm{CAD}$ is no longer enough and most often does not permit complete or adequate evaluation. As far as certain products are concerned, there is no substitute for the ability to touch, manipulate, validate or operate a prototype (design or feasibility model).

It is possible today, by additional treatment of physical models produced through a rapid prototyping process, to go further, indeed to design the process for producing standard parts (models or intermediate parts that can be used in industrialization such as EDM electrodes or mold cavities).

Rapid prototyping (Binstock, 1994) (Bernard, 1995) makes it possible to directly create the physical model from the digital CAD model within twenty-four hours, or at the most a few days. The economic advantage is obvious. Moreover, rapid prototyping provides guidance for design choices (modification, alternatives. etc.) by making a model available much earlier in the project planning stage.

After a short description of rapid prototyping ways and means, we propose some parametric models and present some methodological results on mechanical and jewelry applications.

\section{RAPID PROTOTYPING: WAYS AND MEANS}

The aim of rapid prototyping is to produce a physical model in record time, at the least possible cost and using a minimum of tooling. With this method it is possible at any time, to try out different alternatives in parallel with a view to choosing the best, to validate the chosen approaches as quickly as possible to minimize the number of iterations, to acquire physical and experimental data as a basis for moving the project forward and to have a concrete basis for discussion for the concurrent engineering project teams.

During the past years rapid prototyping has been used mainly to produce form prototypes (needed for validating shapes and as a basis for communication (aesthetic design, part geometry, mechanical design, etc.), functional prototypes (in 
a material close to the real product, any type of process, needed for validating the required functions and assemblies) and industrialization prototypes (needed for validating and determining the feasibility of the production processes, in the real material (or similar) and process similar to the final one).

Rapid prototyping means are well known as techniques based on layer-by-layer manufacturing in order to produce any shape without tooling. The main layer-bylayer processes available in industry account for over 2100 installations around the world including about 80 in France (mainly 3D Systems, EOS, Helisys, Stratasys, DTM, Cubital, Sanders, BPM, Soligen and Stratoconception processes). We shall not go into the detail of each process here since these were covered in many publications (AFPR, 1996).

The essential need in the field of rapid prototyping is always a numerical definition of parts, from CAD modeling or reverse engineering.This approach applies to digitizing and duplicating single objects produced by hand, to comparing and tracing modifications made to a physical model, to designing, developing and producing tooling, to duplicating or even generating a range of parts directly from the file of points (STL file, CLFile), and to following up all the iterations in the design cycle.

Some CAD software packages now offer modules for dealing with point clouds to assist the draughtsman in creating surfaces (Bernard, 1997).

Rapid prototyping also includes post-treatments (for plastic and metallic parts) (Yeung, 1996).Plastic parts can be obtained either directly on layer manufacturing machines (stereolithography, SLS, BPM, FDM), or using vacuum casting in silicone rubber molds, or through the use of metallized prototype molds which are used for the injection of a small batch production. Metallization processes can also be used for EDM electrodes obtention.Prototype metallic parts are mainly produced or raw formed by foundry (Shell, block, and sand casting) or by rapid prototyping processes.One can notice the ability of direct manufacturing of Croning type cores, using SLS process (EOSSint, 1996).

Globally, the development of such processes illustrates the industrial demand for rapid tooling. The ability of rapid tool manufacturing implies particular functions and models which have to be generated and managed, when taking into account the specific technological rules of each technology and the capability to interact between parts and tools (PROD, 1997)

\section{PARAMETRIC MODELLING IN RAPID PRODUCT DEVELOPMENT}

As shown before, the development of a mechanical object widely uses CAD, reverse engineering and rapid tooling techniques. The first example below illustrates the interactivity between the different data models, point clouds, reference models, parametric models, etc. (Dionigi, 1996). 
The objective of this work was to define a parametric model of a tile and to undertake the acquisition of points belonging to its basic shapes (Figure 1) to modify some kinds of tiles and to compare them to a reference model in order to avoid a drift of geometry at one time. It is indispensable to have CAD models of all kinds of produced tiles, and to decrease the stocking of pattern models.

Positive results were obtained on both the modeling and the methodology level. A complementary constraint concerned the imposed CAD platform, Pro/Engineer (PTC_PE, 1996).

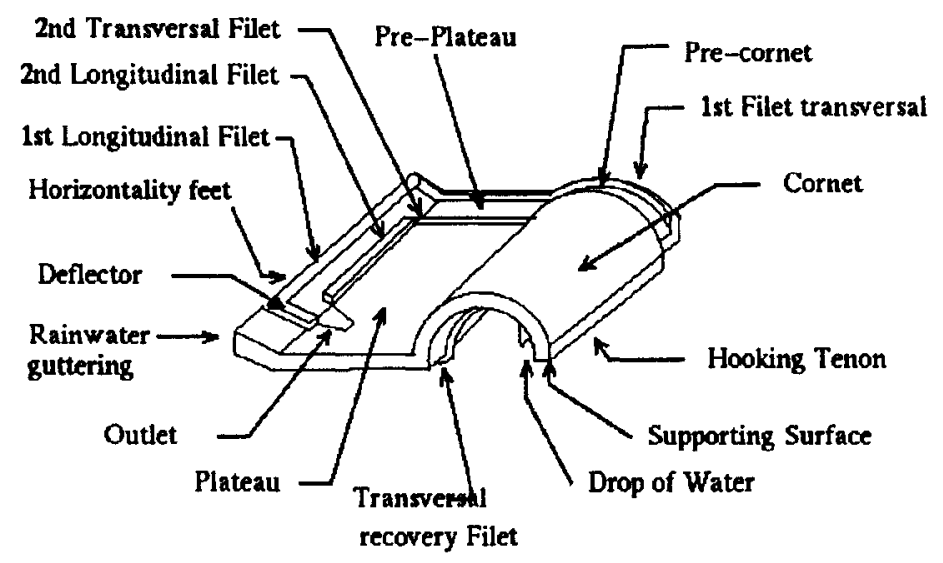

Figure 1 Schema of a roofing tile

\subsection{Functional parametric approach}

Research work related to a functional approach of object modeling are mainly relative to kinematic connections (Rieu et al., 1994) and mechanical components (Chambolle, 1995).

In our approach, we do not design a part from its external forms, but re-build it from its trade-parameters.

The tile is a complex technical object related to a particular context both in elaboration and in utilization. The norms about tiles are solely relative to the process of tile manufacturing; we cannot use them for tile definition. So, it was indispensable to work with professionals of this trade in order to analyze with them the functional forms and constraints.

Fortunately, the part is well known and it can be modeled from its tradeparameters. The expertise gives us the functional surface decomposition.

The first question for parametric model elaboration is the choice of parameters, which means defining tile surfaces and constraints between the surfaces.

In the following paragraphs, each step of our approach to obtain the parametric design model and process of tile is explained in detail. 


\section{Research of parameters}

We applied a functional analysis in order to determine forms of tiles and their constraints as the consequence of functional product needs.

We are not going to stress on the methodology used and the same is available in (Dionigi, 1996). The relationships of the tile had to be defined together with its external context, its functions, and the interactions between functions and the constraint sub-functions they imply. The consequence of these functions and subfunctions is the definition of functional constraints of forms constituting the tile. For example, in addition to classic functions of inclemency and aggression protection, tiles have to be piled up in order to allow their transportation and their manipulation. One understands the interest to constrain the definition of some external and internal forms. So, applying this analysis on our functional surface decomposition, we are able to determine all the constraints for each functional surface (complementary forms, distances, etc.).

Thus, in order to reach our aim of determining the tile parameters, we had to define for every functional surface, the parameters needed to create it, and the constraints it has to respect. Then, we are able to propose a functional generic parametric model of the tile.

\section{Functional parametric model}

The proposed model is based on an object-oriented representation.

Each functional form of the tile is described by:

- its name as identifier (eg. : cornet)

- its physical description (eg. : rounded shape constituting a major element of the morphology of the tile)

- its site on the tile, in reference to a predefined orientation of the tile (ex : right part of the tile)

- its functions (eg. : piping of water; recovery right/left; recovery over/under; horizontality of piling)

- a parametric construction with dimensions accessible or allowable from the external forms of the tile (in fact, accessible by digitizing)

- a design process, here in the environment of Pro/Engineer (eg. : definition of plan profiles and construction of the general form of the cornet by the function "smooth protrusion" with respect to limit constraints)

- its relationships with the other functional forms like constraints of form, dimensions, etc. (e.g., condition of compatibility between external and internal profiles for piling; condition of connection with the plateau; limitation along length direction). In general manner, these relationships show trade as well as the next following constraints : identical to; extremity of; parallel to; defined by; pasting to; confuse with.

Complementary relationships, that we have called "option of forms", were introduced at the global level of the tile model (cornet more longer than the plateau, tile squared, etc.). The update of the library of global options allows for the integration of new tile models. 


\section{Implementation of the model}

The model was implemented under Pro/Engineer. The design process is directly dependent on parametric possibilities and functions of creation of available forms under Pro/Engineer. Especially, basic forms (cornet, plateau) are most often built using a protrusion function, and filets and tenons are constructed by the deformation of basic forms.

The user can choose and modify each parameter, directly on the screen with the mouse or using the software interface that makes elementary parameters and relationship tables (external and internal parameters) accessible.

Thus, from the basic model and from the specification of the "option of forms", the designer has directly a model that will be completed and finished according to final specifications and additional local details. This last phase is essentially relative to the fine definition of internal forms that, in case they exist, are specific to each model.

In the environment of Pro/Engineer one can undertake a rapid simulation of jointing (installation on roofing) and of piling (stocking). One can thus pilot some global dimensions by a constraint of the number of tiles to the squared meter.

In conclusion, we propose a parametric CAD model and apply it to a roofing tile. First we undertake a functional approach of the tile and build a parametric model of the tile, sufficiently generic for defining a great number of its forms solely from functional specifications (dimensions, relationships, sections, etc.), the general topology being automatically managed by Pro/Engineer.

Finally, the parametric model allows us to produce different prototypes of tiles, using stereolithography, and different prototypes of dies for tile forming using three axis NC machines.

The introduction of parametric data can be undertaken by the user directly on the CAD workstation, or via Pro/ScanTools module (PTC_PST, 1996), by digitizing of forms on the physical model (Figure 2).

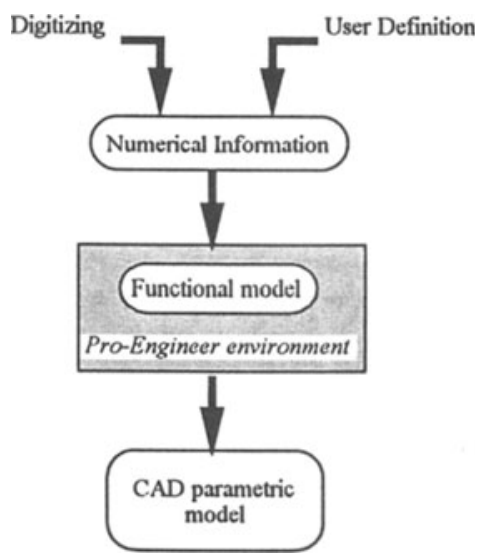

Figure 2 Two ways for parametric model definition 


\subsection{Integration of digitizing entry in the design phase}

The introduction of parametric data is undertaken by the user directly on CAD workstation, or via the Pro/ScanTools module, by the digitizing of parametric curvilinear elements on the physical model. The main difficulty is to determinate these curves from the point clouds obtained by digitizing. For the scanning, we have used a line laser sensor (Kréon, 1996) on CREATE platform (CREATE, 1993) (European Rapid prototyping Center for Assistance, Transfer and Experiments.

The different steps of reverse engineering process are described on Figure 3.

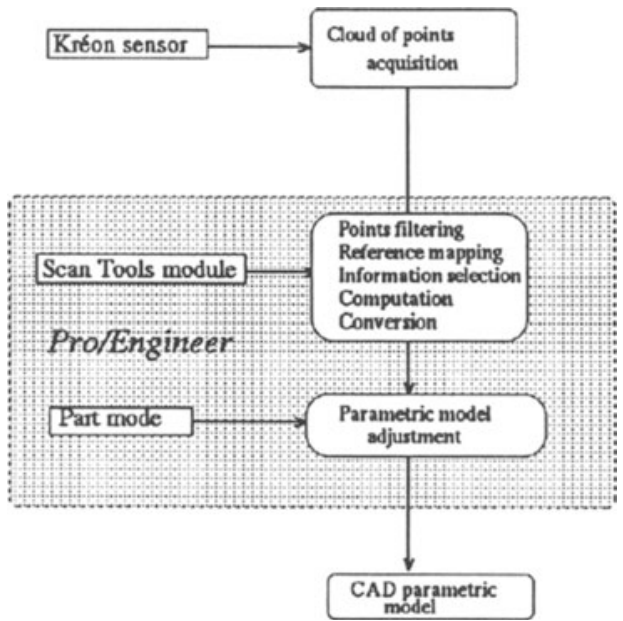

Figure 3 Different steps of reverse engineering integration for tile

One of the main problems was to fit the reference systems of the physical part with those of Pro/Engineer. Then, we were able to introduce scanned parametric curvilinear elements into the generic parametric model of tile.

\section{INTEREST OF PARAMETRIC MODELLING FOR JEWEL DESIGN}

More generally, today the field of artistic creation is directly concerned by the evolution of the design methods and rapid manufacture of new objects. Many artists and creators use computers to program geometrical forms referring to complex combination criteria and generation (Becker, 1995) (Lavigne, 1995). If the shading of results is easily available, the unique solution for the manufacturing of volumic objects is layer-manufacturing.

Concerned by the creation of aesthetic and artistic objects, several companies that create jewels have requested case studies for digitizing, layer-manufacturing of prototypes and validation in the foundry (Suret, 1996). 
In the following paragraphs we are going to present several examples that, completely or partly, have been realized by the CREATE platform during the last years.

\subsection{Creation of normalized finger diameter rings from a unique master}

In this study the goal is to decline all normalized finger diameter rings from a master created in the traditional manner by the jewel designer from the view of its manufacture in a gold foundry.

After an analysis of the shapes (identical motives connected by round blendings of imposed value), we operated the 3D scanning of the master on a Surveyor LD 500 integrated digitizing environment by using a laser point sensor (Laser Design, 1995). As the acquisition of a motive cannot be undertaken in one position, a setting was realized. First we accessed in polar to external forms, thanks to the fourth axis of the Surveyor, then, we recaptured in cartesian scanning the lateral forms rejoining the interior of the two sides. Then it was necessary to adjust the three point clouds with respect one another. In order to do that, the elements of reference (cylinders and planes) were machined on the used setting and scanned at the same time as the forms of the master for each position of the setting on the digitizing machine.

Then one identified the parameters of repositioning (rotations and translations) from the elements of reference and one applied them to the complete point clouds. Then it was classically necessary to filter noises coming from the machine of acquisition and to preserve only significant points for the external surface construction of the ring in the $\mathrm{CAD}$ environment. In the present case the next phase was undertaken on the Strim-RDS module (Strim-RDS, 1996).

The first step was to construct elementary surfaces of the ring from the point clouds, while already thinking of the future declination corresponding to normalized diameters of fingers. Indeed, the designer required that the width not vary in the same manner as the two other dimensions, while being able to preserve the same number of motives and the same weight of the jewel.

Parting from the surfaces of the external form one determined those that will limit the material thickness. Thanks to this thickness parameter, one could postpone the variation of the mass of the ring until the moment of the creation of the offset form constituting the interior. The global difficulty was to integrate the totality of constraints fixed by the designer to the chosen final solution.

The complexity of this phase of construction of the complete surface model of a ring depends mainly on the judicious choice of the parametric decomposition used and the methodology used with Strim-RDS tool.

It is difficult for the designer to appreciate the numerical result displayed on the screen of the workstation, we validated using a stereolithography manufacturing in order to have an object to compare to the basic master. Some modifications were necessary on the $\mathrm{CAD}$ model, essentially on the curve of the motive constituting the periphery of the ring. 
After validating the created numerical representation made on a new part in stereolithography, the phase of diameter declination of fingers was undertaken. At this level the pertinence of the choice of the initial topology of surfaces and the methodology of construction of the first model had considerably facilitated the work.

After the construction of the ten models, STL files were created via the corresponding interface and prototypes manufactured in stereolithography on the SLA250 3D Systems machine of the CREATE.

These prototypes were then exploited during the final foundry phase, to create industrial tools necessary for the gold foundry.

The whole process brought about a gain in terms of cost and time (about $40 \%$ ) compared to traditional methods used until then, despite the prospective character of this study.

\subsection{Towards an efficient declination of a range of jewels in the field of figurines}

The problem of family declination of jewels concerns basic composite figurines that are either porters of stones or inserted on rings with known diameters and thickness. In this case, one undertakes the scanning of a master corresponding to a particular model (stone diameter or characteristics of a well-known ring) and in most of the cases one can apply a direct homothety to the scanned model. Subsequently, the transition to a CAD surface model is not indispensable. Indeed, we have a direct interface for STL format from point clouds.

In the future, the declination of a line of jewels based on figurines (celebrities, animals, objects, etc.) is oriented to a parametric definition of a CAD model reconstructed from point clouds. In order to do that, one works on a functional analysis of forms, with the placement in obviousness of definition parameters intrinsic of forms, variables of positioning and laws governing the global topology of the family of jewels that has to be elaborated.

One can thus construct a library of objects of parametric parameters, offering the possibility to preserve the coherence of the model in the case where, for example, the position of an arm, a leg, the chest or that of the head changes.

The main difficulty of this approach comes from the appropriate definition of the parameters for the reconstruction of the models.

This parametric way is explored in parallel of works relative to the automatic line determination characteristic of clouds of scanned points, in order to be able to make the quasi-automatic definition of a division of constructed surfaces, and the parameter definition.

\section{CONCLUSION}

Rapid prototyping is a set of tools for computer aided design and industrialization of a new product. Nowadays, it is widely used for the concurrent engineering approach.In this paper, we have exposed applications in the field of product integrated design. 
A functional parametric model was presented using functional analysis of a technical object (tile). This model, exploited in the environment of Pro/Engineer, is a real aided-design system for tile definition for the designer, also using the reverse engineering "closing loop" through the acquisition of significant elements by $3 \mathrm{D}$ scanning of a physical part. The model remains open and can be personalized thanks to an object structure, relationships between internal parameters (dimensions) and external ones, and constraints of forms.

We also presented applications of parametric modeling for jewel design.The reengineering and the evolution of models are greatly favored, due to the fact that the parametric model and conditions of existence of each form.

The rapid product development implies the installation of relevant and coherent data structures, directly driven from some relevant functional parameters.

Some particular efforts on global data modeling and integration of all the described functions are ongoing today, mainly based on the STEP Standard (Mony, 1996) (IMS-RPD, 1997).

\section{REFERENCES}

AFPR (1996), 5th European Conference on Rapid Prototyping, French Rapid Prototyping Association, Paris

Becker, A., Bernard, A. (1995), Numerical design of art objects and jewels, 4th European Conference on Rapid Prototyping, Paris

Bernard, A. (1995), Rapid prototyping, International ISTA Conference, Barcelone Bernard, A. (1997), State of the art of reverse engineering, MICAD 97, Paris

Binstock, L. (1994), Rapid Prototyping Systems : Fast Track to Product Realization, Society of Manufacturing Engineers

Chambolle, F. (1995), Functional design of cutting tools, Graduate report, DEA Production Automatisée, ENS Cachan, France

CREATE (1993), Official inauguration of the European Rapid prototyping Center for Assistance, Transfer and Experiments, École Centrale Paris, France

Dionigi, O. (1996), Modelization of functional shapes of mechanical parts for reverse engineering application on a tile, Graduate report, DEA GSI, Ecole Centrale Paris, France

EOSSint (1996), Selective laser sintering process of Croning type sand, Technical documentation, EOS, Germany

IMS-RPD (1997), European and International proposals on Rapid Product Development

Jacobs, P.F. (1992), Rapid Prototyping and Manufacturing ; Fundamentals of Stereolithography, SME

Kréon, (1996) Reverse engineering demonstrations and applications, Kréon Industries, France

Laser Design (1995), Surveyor LD 500 and DataSculpt user manuals, Laser Design Inc, USA

Lavigne, C. (1995), For a new approach, the meeting of artist and engineer, 4th European Conference on Rapid Prototyping, Paris 
Mony, C. (1996), Integration of Rapid Product Development Technologies information models using STEP, Rapid Product Development Technologies, Proceedings of SPIE International Conference, Boston, volume 2910, 156-165

PROD project (1997), Rapid Production of Consommable Tools, project proposal, Ecole Centrale Paris, France

PTC_PE (1996) Generalities - Pro/Engineer Release 17.0, Parametric Technology Corporation, USA

PTC_PST (1996) Pro/ScanTools User's Guide Release 17.0, Parametric Technology Corporation, USA

Rieu, D., Constant, D., Nguyen, G.T. and Tollenaere, M. (1994) Integrated design of kinematic connections, IFIP conference, Valenciennes, France

Strim-RDS (1996), Technical documentation, MatraDataVision, France

Suret, S., Bernard, A., Bocquet, J.C. (1996), Using rapid prototyping for new products development : application to jewellery design, Rapid Product Development Technologies, Proceedings of SPIE International Conference, Boston, volume 2910, 216-225

Yeung, M.K. (1996), Rapid tooling : a step change in mold manufacturing, Rapid Product Development Technologies, Proceedings of SPIE International Conference, Boston, volume 2910, 176-186

\section{BIOGRAPHY}

A. Bernard was graduated in 1982 at ENS Cachan. He contributed to LURPA laboratory, with Prof. Bourdet, since 1983 and obtained his $\mathrm{PhD}$ in 1989, on 3D feature-based manufacturing of forging dies. As an assistant professor, he worked from 1990, for six years, with Prof. Bocquet in Ecole Centrale Paris on product, technology and process modeling. He also created, in 1993, the rapid prototyping and reverse engineering platform of Ecole Centrale Paris, the CREATE. He is the vice-president of AFPR (French Rapid Prototyping Association). Since September 1996, he is Professor in CRAN laboratory in Nancy.

M. Véron is professor and has been the chairman of CRAN laboratory (Research Center for Automatic Control of Nancy) during the last six years. He also manages an integrated $\mathrm{CAD} / \mathrm{CAM}$ and production engineering platform (AIPL) used by the students of the university. He is also the general secretary - treasurer of CIRP (International Institution for Production Engineering Research). 